\title{
The effect of farmer nutrition schools on household food production and women's dietary diversity in Bangladesh
}

\section{Lidan Du ${ }^{a}$, Timothy Williams ${ }^{b}$, Aaron Hawkins $^{c}$, Sarah McClung $^{\mathbf{b}}$, Teemar Fisseha $^{\mathbf{b}}$, Heather Danton ${ }^{b}$}

a The SPRING Project (Strengthening Partnerships, Results, and Innovations in Nutrition Globally)/Helen Keller International, Arlington, VA, USA

b The SPRING Project/JSI Research \& Training Institute, Inc., Arlington, VA, USA

${ }^{c}$ Save the Children, Colombo, Sri Lanka.

Correspondence to Timothy Williams (tim_williams@jsi.com)

\section{Funding}

The SPRING project and this research were funded through the United States Agency for International Development (USAID) under the terms of the Cooperative Agreement AIDOAA-A11-00031, SPRING), managed by JSI Research \& Training Institute, Inc. (JSI).

\section{Acknowledgement}

We thank Gwyneth Cotes of SPRING for inputs to an earlier draft of this paper, Wahid Quabili of IFPRI Bangladesh for providing the data points from BIHS, and Hillary Murphy of SPRING for editing and formatting assistance. 


\section{Abstract}

Introduction: The nutrition situation in Bangladesh has improved substantially over the last decade. Nevertheless, dietary diversity of women of reproductive age continues to be low. In addition, global evidence on the effect of nutrition-sensitive agriculture interventions on dietary diversity is still developing. The Strengthening Partnerships, Results, and Innovations in Nutrition Globally (SPRING) project implemented a Farmer Nutrition School (FNS) intervention in two divisions (regions) in Bangladesh from 2012 to 2016. FNS targeted pregnant women and mothers with children less than two years of age (PLW) in the lowest two wealth quintiles. FNS was based on three well-established, evidence-based strategies--the essential nutrition and hygiene actions, the homestead food production approach, and farmer field schools.

Methods: This study aims to assess the effect of SPRING's FNS program on women's dietary diversity, measured by a women's dietary diversity score (WDDS) based on the nine-item FANTA/FAO scale. We followed up one cohort of women who participated in FNS beginning in November 2014. We undertook a two-stage sampling design to identify these women. First, forty-four FNS groups were randomly selected from the 2,560 FNS that were planned to be implemented during 2014-15. Second, 10 women were randomly drawn from each of the 44 FNS groups, which were scattered across 38 upazilas (sub-districts), 19 each in Barisal and Khulna Divisions. Sample size was calculated to detect a 0.25 effect size in the WDDS with a power of $80 \%$ and confidence interval of $95 \%$. Three phases of surveys were conducted: before the FNS began (Phase 1-P1, November 2014), immediately after the completion of the FNS (Phase 2-P2, July - August, 2015), and one year after (Phase 3-P3, July 2016). A total of 386 women completed all three surveys. Data analyses included descriptive statistics and t-tests. Statistical significance is tested by the adjusted Wald test with a 95\% confidence interval.

Results: The percentage of FNS participants' households that produced vegetables, poultry and fish significantly increased between P1 and P2, from $43 \%$ to $95 \%(\mathrm{p}<.05)$, from $75 \%$ to $87 \%(\mathrm{p}<.05)$, and from $48 \%$ to $67 \%(p<.05)$, respectively. The increases were more or less sustained at $\mathrm{P} 3$, at $86 \%, 92 \%$, and $63 \%$, respectively. Additionally, the mean numbers of vegetable types grown and of chickens reared increased significantly from P1 to P2 $(\mathrm{p}<.05)$ and were sustained at P3, from 1.3 to 4.9 to 4.6 , and from 5.3 to 7.9 to 8.4, respectively. The mean WDDS significantly increased from 3.9 to 6.0 between P1 and P2 ( $<<.05)$, and was sustained at 5.6 at P3. The proportion of women who reported consuming items promoted in FNS, such as eggs, vitamin-A rich fruits and vegetables, and flesh foods also increased significantly.

Conclusions: The FNS approach, which supports agriculture interventions with an explicit nutrition objective, generated rapid and sustained improvement in food production and dietary quality. This study suggests that a program design based on local contexts with moderate management support and minimum material inputs can have a powerful effect on women's dietary diversity within a short time. Furthermore, with community involvement and ownership in a context like rural Bangladesh, the improvements can be sustained, at least for one year. While this cohort study did not have a built-in control group, the magnitude of the increases in production outcomes and WDDS among FNS participants, coupled with much smaller or no increases at the population level over even longer time periods, suggested that these results were probably largely due to the SPRING FNS intervention.

Key words: Farmer Field School, nutrition, Bangladesh, Feed the Future, women, dietary diversity 


\section{Introduction}

Despite improvements in nutrition in Bangladesh over the last decade, the nutritional status of young children and women of reproductive age continues to be a public health concern. The most recent Demographic and Health Survey (DHS) published in 2014 reported improvements since the 2011 DHS, yet the stunting and wasting rates for children under five years of age remained high at 36\% and 14\%, respectively, and 31\% of ever-married women aged 15-49 years were undernourished (BMI <18.5) (DHS Bangladesh 2011, 2013).

Dietary diversity is a proxy measure for nutrient adequacy of the diet of individuals. Variety in an individual's diet is associated with the intake of essential nutrients and is especially important for people living in resource-constrained settings where the diets are largely based on starchy staples (Ruel 2003). Evidence suggests that women's dietary diversity in Bangladesh is low, even among pregnant women, who have greater nutritional needs. A 2013 study (Shamim et al. 2016) of the diets of pregnant women in Southern Bangladesh found that the mean WDDS was 4.28 (out of nine-point food groups score). A 2015 survey (Sinharoy et al. 2018) of women aged 15-40 in Habiganj District of Eastern Bangladesh reported a mean of 3.9 (out of 10 food groups as delineated in the newer Minimum Dietary Diversity - Women (MDD-W) indicator (FAO and FHI 360 2016).

Since the 2013 Lancet series on nutrition discussed agriculture programs’ ability to improve nutrition and pointed to the need for stronger evidence, researchers and implementers have investigated nutrition-sensitive agriculture programming to further contribute to the evidence base and operationalize the most promising approaches (Ruel and Alderman 2013). The United States Agency for International Development (USAID) launched its Feed the Future initiative (USAID 2010) to simultaneously address global hunger and poverty. Under the initiative, multiple projects were designed and implemented in 19 focus countries integrating nutrition into more traditional agricultural programming. Agricultural market systems development and homestead food production are two strategies that have commonly been promoted as nutritionsensitive means of increasing household and individual dietary diversity.

The Strengthening Partnerships, Results, and Innovations in Nutrition Globally (SPRING) project was a USAID-funded global nutrition project (2011-2018) with a mandate to develop technical guidance to improve the effectiveness of agricultural activities on nutrition and contribute to the evidence base. The SPRING project implemented the Farmer Nutrition School (FNS) intervention in 40 upazilas ('sub-districts') of Barisal and Khulna divisions (regions) in the Feed the Future Zone of Influence (ZOI) ${ }^{1}$ in Bangladesh from 2012 to 2017. FNS targeted pregnant and lactating women (PLW) and women with children under two years of age, who belonged to the lowest two wealth quintiles within their communities. FNS was based on three well-established, evidence-based strategies, namely: the homestead food production approach, essential nutrition and hygiene actions, and farmer field schools.

Homestead Food Production (HFP) is an approach intended to increase dietary diversity through

\footnotetext{
${ }^{1}$ The Feed the Future zone of influence in Bangladesh comprised the southern part of Dhaka division and all of Barisal and Khulna divisions. There are 8 divisions in Bangladesh; these 2.5 divisions therefore represent a little over a fourth of the total geography of the country.
} 
support to small home gardens. HFP uses both supply of inputs and agricultural education combined with nutrition education that encourages women to consume the nutrient-rich vegetables and fruits that they produce in their gardens. The HFP model targets women, and focuses on their nutrition education and behavior change (Iannotti, Cunningham, and Ruel 2009). The HFP model evolved over time with increased emphasis on nutrition and hygiene practices. Studies have found that the newer enhanced-HFP (E-HFP) approach improved women's intake of select nutrient-rich foods and dietary diversity, among other nutrition outcomes, in experimental trial and program settings in Burkina Faso and Nepal (Olney et al. 2016; Dulal et al. 2017).

The Essential Nutrition Actions (ENA) refers to an integrated package of key preventive nutrition actions targeting the first 1000 days of a child's life - from conception through the second birthday of the child. The guide Essential Nutrition Actions: Improving Maternal, Newborn, Infant and Young Child Health and Nutrition released by the WHO in 2013 summarizes recommendations of nutrition-specific and nutrition-sensitive actions to improve maternal, infant, and young child nutrition when taken to scale (WHO 2013). The ENA framework is considered to be a global best practice and is used around the world engaging health service providers and other actors to improve the nutritional needs of women and children. In 2015, a new package of ENA tools was enhanced to include Essential Hygiene Actions (EHA) which link improved nutrition with handwashing, food hygiene, safe water, and animal hygiene (CoreGroup 2015).

In addition to HFP and ENA, SPRING utilized a third proven behavior change mechanism - the Farmer Field School (FFS) approach developed to disseminate practical information to farmers. FFS was first developed by the Food and Agriculture Organization (FAO) as an alternative to mainstream agricultural extension systems that had a heavy reliance on chemical inputs in Southeast Asia in the late 1980s (Braun and Duveskog 2008). The FFS approach is based on adult non-formal education, using peer-to-peer support, and a prolonged, two-year 'learning by doing' approach. FFS uses simple or traditional technologies and encourages farmers to compare various techniques that work best for them (FAO 2018).

Globally, the evidence base with regard to the effects of household food production on maternal and child nutrition has been growing since the last review (Girard et al. 2012). In addition to the findings based on the E-HFP approach, agricultural programs that included FFS and other components, when integrated with nutrition education, were also found to be able to contribute to improved nutrition outcomes such as increased dietary diversity for women or children in few Asian and Sub-Saharan African countries (Doocy et al. 2017; Reinbott et al. 2016; Kuchenbecker et al. 2017). This paper aims to contribute to this global evidence base by reporting an observed effect on women's dietary diversity following participation in FNS, an integrated learning and practice intervention, which included agriculture, nutrition, and hygiene components. These findings may inform future research and program design worldwide.

\section{Methods}

This article describes the extent to which the SPRING FNS program affected the changes in 
WDDS, one of several key program outcomes, among a cohort of participants between 2014 and 2016 in Barisal and Khulna divisions of Bangladesh. We summarize the details of the SPRING FNS interventions, study design, data collection, and statistical analyses below.

\section{Farmer Nutrition School Interventions}

The FNS program was developed to help community members and groups improve nutrition practices through a multifaceted, intensive program of learning sessions focused on food production, hygiene, and nutrition. The program integrated and streamlined ENA and EHA into agriculture training modules to promote production and consumption of nutrient-dense vegetables, and animal source foods (fish, poultry, and eggs). The plant varieties to be produced on homesteads were carefully selected through looking at the local nutritional profile and nutrient-dense foods that were also most readily available in the SPRING project area. SPRING also conducted focus group discussions at the onset of program to determine which vegetables and fruits would be most acceptable to the community. Vegetables and fruits identified included sweet gourd, bottle gourd, knolkhol, radish, red amaranth, stem amaranth, kangkong, Indian spinach, okra, bitter gourd, tomato, papaya, ash gourd, country bean, and yard long bean.

The full FNS program had 18 sessions, grouped into three modules as listed in Box 1. Although listed as separate sessions, key messages and practices of ENA and EHA were actually incorporated into each session to reinforce and encourage women and their families to adopt improved practices in health and nutrition as well as HFP, on an ongoing basis.

\section{Box 1. Farmer Nutrition School sessions by Modules}

Module 1: Vegetable Gardening

1. Homestead Space Planning and Vegetable Bed and Pit Preparation

2. Sowing Seeds and Planting Seedlings in the Bed and Pit

3. Crop Cultivation Operations, Soil Health Management, and Compost/Manure Preparation

4. Integrated Pest Management

5. Seed Production, Collection, and Storage

6. Essential Nutrition Actions and Essential Hygiene Actions

Module 2: Native Chicken Rearing

1. Basic Techniques for Local Chicken Rearing

2. Improved Housing and Laying Hen Management

3. Broody Hen Management

4. Chick Rearing Management Session

5. Health Management and Egg or Meat Consumption

6. Essential Nutrition Actions and Essential Hygiene Actions

Module 3: Pond-Fish Culture

1. Understanding Improved Fish Culture Techniques

2. Preparing the Pond for Fish Stocking

3. Fingerling Transportation and Stocking

4. Supplementary Feeding, Pond Fertilization, and Liming

5. Health and Growth Management and Fish Harvesting

6. Essential Nutrition Actions and Essential Hygiene Actions 
The sessions were carried out in the form of participatory discussion, questions and answers, demonstration, reflection on practices and lessons learned since the last meeting, and hands-on practice. SPRING Union (a subdivision of upazilas, each consisting of several villages) Facilitators chaired the FNS sessions. They were trained by our upazila coordinators and senior technical officers with backgrounds in fisheries, nutrition, veterinary, and horticulture, who collectively wrote the FNS curriculum. Each session typically lasted for 2-2.5 hours and took place in the courtyard of one FNS participant's household, preferably near her vegetable garden. Some women were accompanied by other female family members on site, to help with childcare.

The SPRING FNS sessions were designed to bring together neighboring resource-poor households with PLW belonging to the lowest two wealth quintiles of the communities. Interested eligible women joined these groups of approximately 16-20 women, who, over the course of nine months, took turns hosting the bi-monthly sessions. At each session, there was one FNS participant who was selected as the day leader; her role was to host the session, help demonstrate during the session and to spend the period of time until the next FNS session following up with other members of the FNS to see if there were any questions or concerns coming from the group.

In addition to the fact that FNS was substantially shorter and more streamlined than the traditional FFS approach and that FNS included nutrition and hygiene components as well as agriculture, FNS also differed from traditional FFS by having fewer inputs. The only material input that was provided by SPRING to the FNS participants was a two-time distribution of a small number of vegetable seeds to get their gardens started. SPRING facilitators brought in a minimal number of teaching aids and food materials for demonstration purposes. It was decided to provide these seeds for two reasons: 1) quality seeds are difficult to come by in many local markets; and 2) the project wanted to ensure that what was planted by the participants would be of the greatest possible nutritional value.

Upon graduation from the 9-month intensive program, all FNS participants received certificates and one woman from their group was selected as their Community Nutrition Champion (CNC) to help keep the practices and messages alive and strong among the group members and within the community. SPRING staff provided one year of infrequent follow-up after graduation (usually once every 2 to 3 months) to see how things were continuing and which, if any, questions had come up since the FNS sessions finished.

\section{Study Design and Sampling}

Over the entire 6-year program, SPRING worked in 40 upazilas and reached 126,255 women through 6,421 FNS groups. Our study focused on one cohort who attended FNS beginning in November 2014. In program year 2014 -15, SPRING planned to enroll 48,000 women into 2,560 FNS groups. We calculated a sample size of 440 women to be drawn from 44 randomly selected FNS groups, to detect a 0.25 effect size in WDDS with a power of $80 \%$ and confidence interval of $95 \%$. We inflated the estimated number by $20 \%$ to account for nonresponse over the two-year follow-up period and some small FNS groups. The calculation was based on mean and standard deviation estimates drawn from the James P. Grant School of Public Health/HKI Food Security and Nutrition Surveillance Program (FSNSP) and the Feed the Future monitoring data sets. 
Enumerators were all experienced data collectors recruited in Dhaka without affiliation with the local communities. Almost all of them had administered survey questionnaires for SPRING partner organizations in country. In addition, they received refresher training and conducted field testing before each round of data collection.

We undertook a two-stage sampling design to identify the women to be included in the cohort. In the first stage of sampling, 2,560 FNS were randomly sorted, which led to the selection of 24 FNS groups from 19 upazilas in Barisal division and 20 FNS groups from 19 upazilas in Khulna division. Figure 1 depicts the FNS sites selected. The second stage of sampling took place in the field. The enumerator obtained a list of names of the participants in each FNS (16-20 per group) from the FNS facilitator, who had listed participants in their order of enrollment when the group was formed. The enumerator selected every other woman on that list to administer the survey questionnaire.

Figure 1. Location of FNS selected

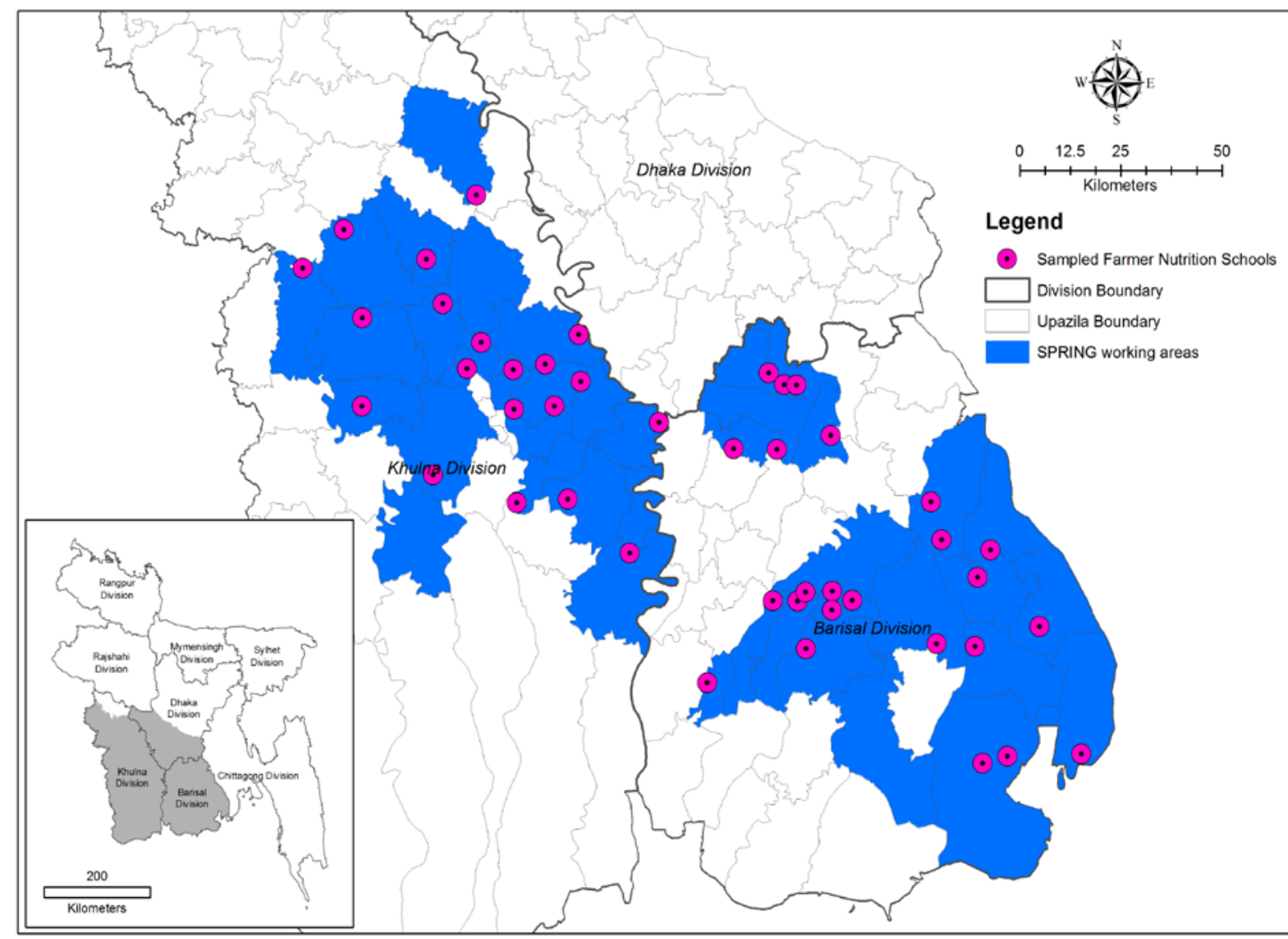

Figure 2 depicts the sample attainment over time from 1) before the FNS training began (Phase 1, November 2014), to 2) immediately after all FNS sessions concluded (Phase 2, July - August, 2015), to 3) one year after Phase 2 (Phase 3, July 2016). There were 386 FNS participants who were included in all three phases of data collection. The major reasons for nonresponse in Phase 2 and Phase 3 were unavailability of respondents despite multiple attempts to contact them and migration to other places. There was no significant difference in socioeconomic characteristics 
between the women who completed all three rounds of surveys as opposed to the 440 women originally selected at $\mathrm{P} 1$.

Figure 2. Sample retained over time and loss of follow up

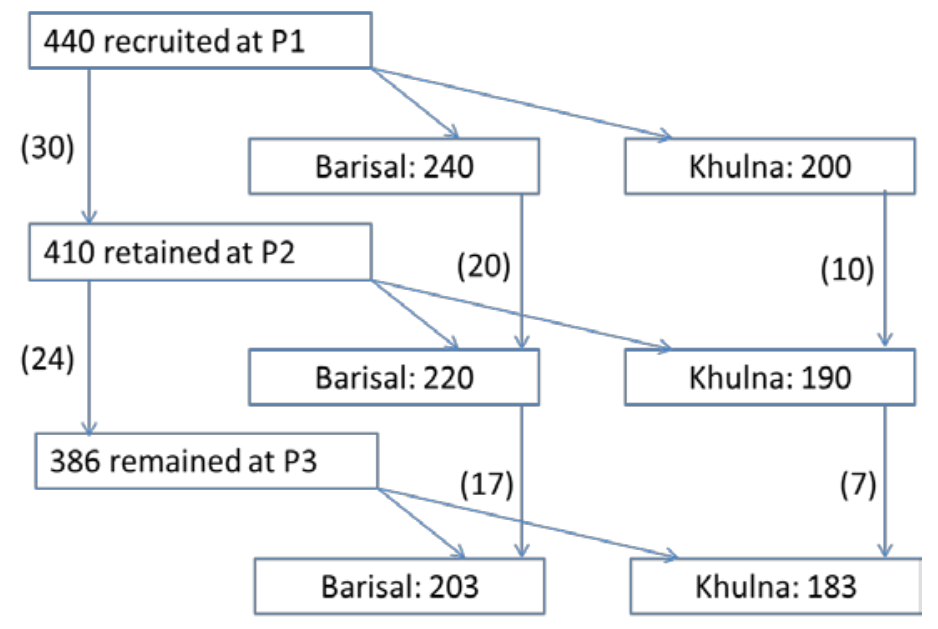

\section{Data Collection}

There were eight sections in the questionnaire: 1) sociodemographic data of women in the cohort, 2) gardening, 3) poultry management, 4) aquaculture, 5) hygiene, 6) women's dietary diversity, 7) child feeding, and 8) nutrition knowledge. The questionnaire was translated into Bangla and tested during the data collection training before Phase 1 . The same questionnaire was used in all three phases and was revised slightly before the second and third phases to ask about seasonal differences in gardening and crop availability.

Ten enumerators collected data in each phase and the majority of them were involved in all three phases. The interviews happened in the individual women's homes. Data collection was supervised by SPRING staff. Completed questionnaires were reviewed by SPRING staff (led by the supervisors) on a daily basis for quality control, and known errors and inconsistencies were corrected, where necessary by revisiting relevant households. Phase 1 data were collected using a paper-based questionnaire and entered into a database by data entry operators using tablet computers; Phase 2 and 3 data were entered directly into tablet computers in the field. Data collection for P1 took place in November 2014, P2 in July-August 2015, and P3 in July 2016. The seasonal difference between P1 and P2/P3 was due to the fact that the total length of the FNS program was 9 months.

\section{Statistical Analysis}

Results are presented using proportions for categorical variables and means for continuous variables. Data analysis was done using Stata (StataCorp, v13.0). Statistical significance is tested by the adjusted Wald test with a 95\% confidence interval. 


\section{Ethical consent}

Before starting the interview, all data collectors took written consent from respondents during the first phase and oral consent subsequently. A clear, easily understandable consent form was designed and included at the very beginning of the questionnaire. Interviews took place in a private area and no financial or other benefits were provided to the interviewees. The protocol was approved by JSI Research \& Training Institute, Inc.’s Institutional Review Board (\#14-028, dated October 29, 2014).

\section{Results}

The results we present here focus on changes in food production, and whether such changes translated into improved diets among one cohort of PLW who participated in FNS sessions. For all indicators reported on in this paper, we provide results from surveys conducted at baseline (immediately before the women began the FNS program), post-FNS (immediately after completing the FNS program), and one year after completion. These time points are referred to as P1, P2, and P3 throughout the report.

\section{Demographic Characteristics}

Table 1 shows some sociodemographic characteristics of the cohort of women included in the study. All women who were enrolled in FNS and selected into the cohort were either pregnant, lactating, or had one or more children under two years of age at the baseline. If there was more than one child under two, the youngest was chosen as the target child. The mean number of years of education was $5.8 \pm 0.15$ years, with a slightly higher but non-significant duration in Khulna $(6.0 \pm 0.21)$ than in Barisal $(5.7 \pm 0.21)$. The mean age of the index child was slightly (not significantly) younger in Barisal (8.3 months) than that in Khulna (8.5 months). Information on the respondents' age was not collected.

Table 1. Baseline characteristics of FNS cohort samples

\begin{tabular}{|l|c|c|c|}
\hline Variable & $\begin{array}{c}\text { Overall } \\
\mathbf{( N = 3 8 6 )}\end{array}$ & $\begin{array}{c}\text { Barisal } \\
\mathbf{( N = 2 0 3 )}\end{array}$ & $\begin{array}{c}\text { Khulna } \\
\text { (N=183) }\end{array}$ \\
\hline Education (\%) & 7 & 8 & 5 \\
None & 24 & 24 & 24 \\
Primary Incomplete & 18 & 21 & 14 \\
Primary Complete & 43 & 37 & 50 \\
Secondary Incomplete & 8 & 10 & 6 \\
Secondary Complete and Higher & $5.8(0.15)$ & $5.7(0.21)$ & $6.0(0.21)$ \\
\hline Mean Number of Years of Education & & & 16 \\
(S.E.) & 18 & 20 & 19 \\
\hline Age of Children <2 Years Old (\%) & 25 & 30 & \\
0 (pregnant*) & & & \\
0-5 months & & & \\
\hline
\end{tabular}




\begin{tabular}{|l|c|c|c|} 
6-23 months & 57 & 50 & 65 \\
\hline $\begin{array}{l}\text { Mean Age of Respondent's Youngest } \\
\text { Child, in months (S.E.) }\end{array}$ & $8.4(0.039)$ & $8.3(0.055)$ & $8.5(0.055)$ \\
\hline
\end{tabular}

\section{Attendance of the FNS Sessions}

SPRING strongly encouraged FNS participants to attend all 18 sessions. As shown in Figure 3, $34 \%$ of women attended all sessions, $56 \%$ of women attended $75 \%$ or more (but not all) of the sessions and $13 \%$ attended less than $75 \%$ of all sessions. Very minor differences were seen between Barisal and Khulna divisions. Differences in participation also did not vary significantly by education or income levels (data not shown here).

Figure 3. Attendance of FNS sessions

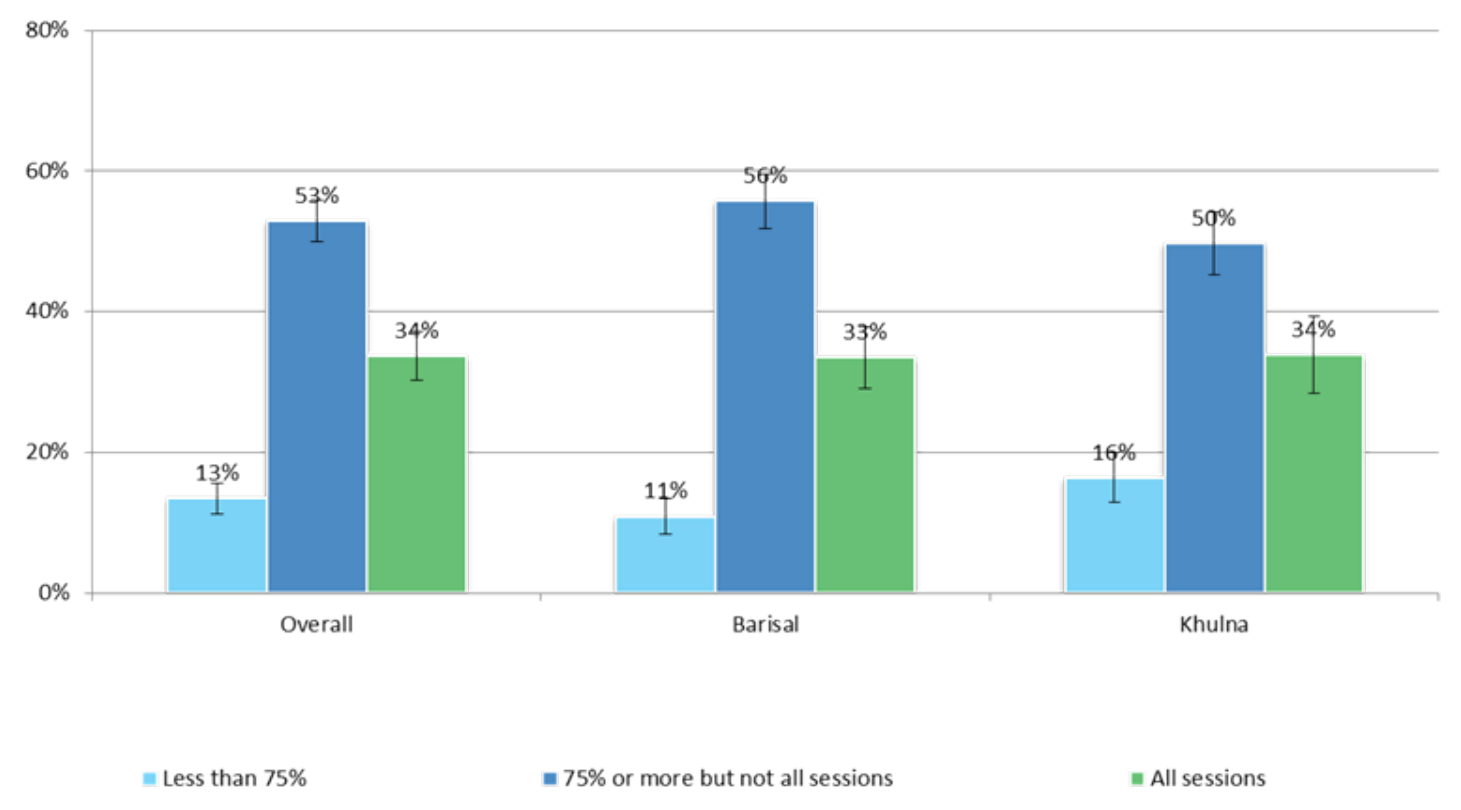

\section{Production outcomes}

A household was recorded as having a homestead garden if fruits or vegetables were produced for household consumption or for sale in the four months before the survey. A household was considered as rearing poultry if the household was currently rearing either chickens or ducks or both at the time of the survey. A household was considered farming fish if they had raised any of the following types of fish: tilapia, indigenous small fish, or a combination of both in the family pond at the time of the survey. Table 2 presents the results in production over time and by division. 
Horticulture At baseline, fewer than half of the women's households were growing their own fruits and vegetables. By the time the nine-month program had been completed, over $90 \%$ had established gardens, and the proportion of households with gardens increased significantly in both Barisal and Khulna Divisions between P1 and P2, as well as in the overall sample. There are some variations between the two divisions in terms of sustained results. The proportion of households that kept homestead gardens dropped significantly from $93 \%$ to $77 \%$ between P2 and P3 for Khulna but stayed nearly the same for Barisal at P2 (97\%) and P3 (94\%).

Table 2. Homestead production over time

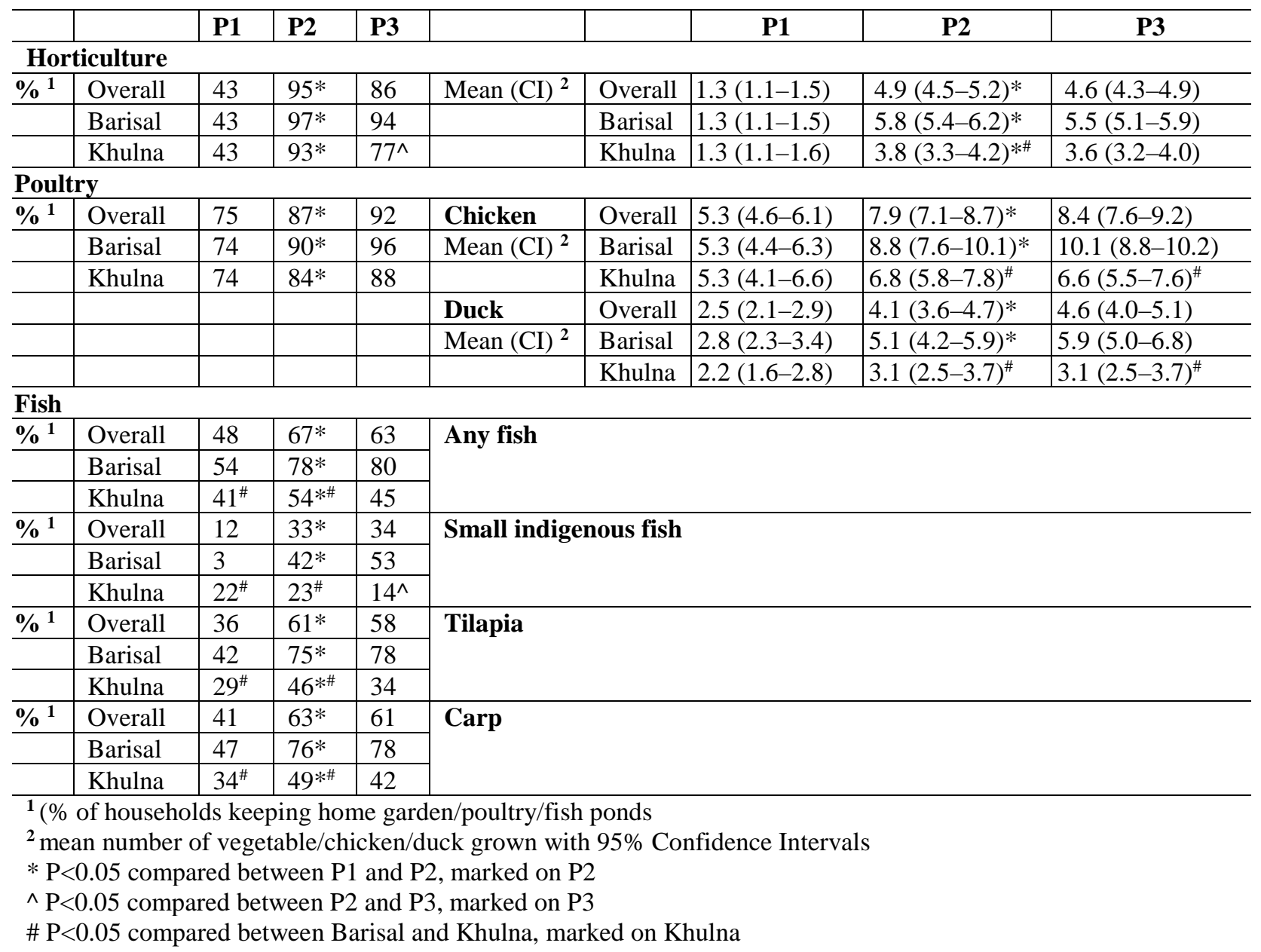

Second, we further explored the number of different kinds of vegetables grown at each phase of the program, as one of the main objectives of the farming component of FNS was to help participants grow a greater variety of fruits and vegetables to improve availability of such foods year-round. Notably, before enrolling in FNS, participants' households in both divisions were growing on average, just over one type of vegetable - 1.3 (CI: 1.1-1.5). The number increased significantly $(\mathrm{p}<.05)$ in both divisions to 4.9 (CI: 4.5-5.2) by the end of FNS at P2, and was 4.6 (CI: 4.3-4.9) at P3. However, there was marked disparity between the two divisions despite the identical low baseline statistics. The mean number of horticultural crops grown in Barisal increased to 5.8 (CI: 5.4-6.2) ( $\mathrm{p}<.05)$ at P2, and dropped only non-significantly to 5.5 (CI: 5.1- 
5.9) at $\mathrm{P} 3$. The mean number grown in Khulna at $\mathrm{P} 2$, although significantly $(\mathrm{p}<.05)$ higher than at P1, was significantly $(\mathrm{p}<.05)$ less than that of Barisal, reaching only 3.8 (CI: 3.3-4.2) at P2, and declining non-significantly to at 3.6 (CI: 3.2-4.0) at P3.

Poultry and Fish The FNS program sought to increase the numbers of households raising poultry and fish, and to increase the quantity of poultry, and variety of fish. The proportion of FNS participants households that reported rearing poultry increased over time, despite that almost three quarters (74\%) of participants' households were already rearing poultry at the baseline for both divisions. The percentage increased to $90 \%$ in Barisal, which was significantly more than that in Khulna (84\%) at P2. And the percentage sustained a year after the completion of the FNS program from P2 to P3 for both divisions. In addition, the average number of heads of chicken and ducks reared per household also increased significantly from P1 to P2 for both divisions. The mean number of birds reared in Barisal was higher than that in Khulna at P2, despite very similar head counts on chicken and ducks kept at P1. The headcounts for both chicken and ducks in Barisal also sustained a year after the completion of FNS program, but the numbers in Khulna did not increase any further. The differences between the two divisions at P2 and P3 were actually statistically significant.

About half of the FNS participants' households were raising fish in their household pond at P1, with Barisal having a significantly higher proportion of participants household farming any fish than that in Khulna (54\% vs. $41 \%, \mathrm{p}<.05)$. The proportion of FNS participants' households engaged in fish farming in Barisal increased to $78 \%$ at P2, significantly more than the $54 \%$ in Khulna at P2 $(\mathrm{p}<.05)$. It continued rising in Barisal from P2 to P3 but the proportion in Khulna actually decreased, both insignificantly. Furthermore, at the baseline, much of the fish farming concentrated on carp. With time, households began farming more tilapia and other small indigenous fish such as Mola, Dhela, Gangetic scissortail, and others. The farming of small indigenous fish - a key promoted practice under FNS - among those participants' households that reported having a fish pond saw a huge increase in Barsal, from 3\% to 42\% between P1 and P2 ( $<.05)$, followed by another 11 percentage-point hike to $53 \%$ at P3. On the other hand, farming of small indigenous fish stayed practically the same between P1 and P2 in Khulna (22 vs. $23 \%)$ and decreased significantly to $14 \%$ at P3 (P <.05).

\section{Consumption and Dietary Diversity outcomes}

Our study used the WDDS, a 9-point food group score, to measure dietary diversity. There is no established cut-off point in terms of number of food groups to indicate adequate or inadequate dietary diversity for the WDDS indicator, because it is "recommended to use the mean score or distribution of scores for analytical purposes." (FAO 2010) Therefore, we computed the mean WDDS (Figure 4), which increased significantly from 3.9 (CI: 3.7-4.1) to 6.0 (CI: 5.8-6.2) between P1 and P2 overall and was nearly sustained at 5.6 (CI: 5.4-5.8) at P3 for the two divisions combined. The WDDS in Barisal was lower than that in Khulna at P1, but not significantly, and at P2, the WDDS in Barisal was significantly higher than that in Khulna, and this significant difference persists at P3. The decline in WDDS from P2 to P3 is very slight in Barisal, with a greater decrease in Khula, but not statistically significant in either case.

The increases in WDDS appear to have been driven mainly by increases in consumption of a few food groups whose increased production was the target of FNS. For example, the proportion of 
women who consumed eggs changed from $27 \%$ to $62 \%$ to $52 \%$ at P1, 2 and 3 overall. The proportion of women who consumed vitamin A-rich fruits and vegetables changed from $15 \%$ to $53 \%$ to $38 \%$ at P1, 2 and 3 . The proportion of women consuming flesh foods changed from $74 \%$ to $90 \%$ to $87 \%$ at P1, 2, and 3 . We also noticed significant increase in consumption of items whose production were not the focus of FNS interventions, such as dairy products. We speculated that this increase was due to the fact that their consumption was consistently encouraged throughout the FNS as part of the nutrition education on dietary diversity, including the benefits of animal-sourced foods in general, which might have been taken up by the FNS participants, who then translated such awareness and knowledge into practice.

Figure 4. Mean women’s dietary diversity score by phase of the study

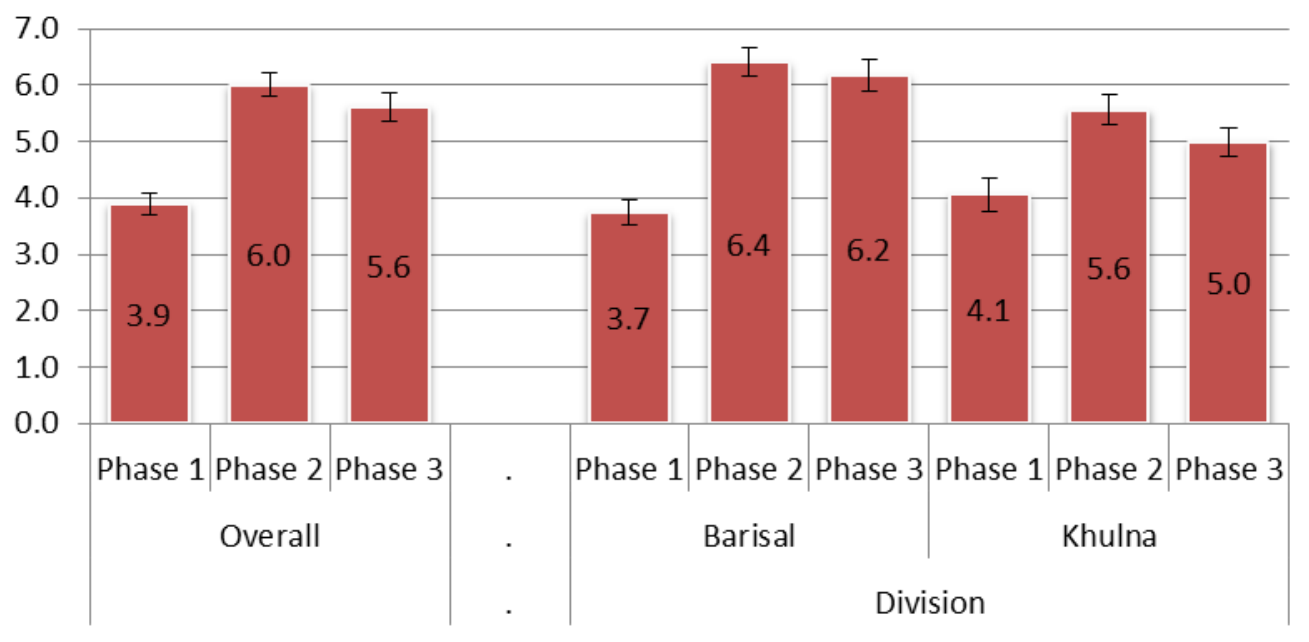

\section{Discussion}

Our program approach was to increase women's dietary diversity via increased homestead production of diverse and nutritious foods year-round, by equipping women with knowledge and techniques on horticulture production, poultry and fish rearing, and nutrition learned in the FNS group. We observed a significant increase in the production and consumption of promoted nutrient-rich foods, particularly for eggs, vitamin A-rich fruits and vegetables, and flesh foods (including meats, fish, poultry etc).

The evidence base on the association between diversity of production and dietary diversity has grown in recent years. One comprehensive review (Jones 2017) of over 20 studies concluded that a positive relationship between agricultural biodiversity on home farms and household dietary diversity exists but with a small magnitude. The review reported an observed threshold effect, which indicates that the dietary diversity will increase with the increase of production biodiversity until the latter reaches or exceeds a certain level (which may be as high as 25 varieties depending on contexts), and that both subsistence- and market-oriented mechanisms could be at play. Given that our participants' households were from the lowest two wealth quintiles and most likely engaged in subsistence production, our results appeared to conform to 
the findings from the systematic review. Specifically in rural Bangladesh, research has found that having a homestead vegetable garden, but not ownership of livestock, was found to be associated with a small but statistically significantly higher WDDS among women of reproductive age (Harris-Fry et al. 2015).

The characteristics of FNS participants and most of the production and dietary diversity features between Barisal and Khulna were very comparable at P1. One notable disparity observed was fish farming practices. In Khulna in P1, a higher proportion of FNS participants' households that had a fish pond farmed small, indigenous fish and a smaller proportion of them farmed tilapia and carp. In P2 and P3, however, there were several significant differences between the two divisions. Although we found general improvement over time in almost all behaviors of interest in both divisions, FNS participants in Barisal consistently achieved higher and better production and dietary results than Khulna respondents at P2. Further, Barisal respondents were also more likely than their Khulna counterparts to retain the improved practices at P3. Many of the differences were statistically significant.

Although it was beyond the scope of our cohort study to identify the reasons behind the differential performances of the two divisions, anecdotal information gathered from discussions with key program personnel in many upazilas of both Khulna and Barisal suggested that both natural resource and program elements could have played a role. In terms of natural resource endowment, Barisal has a lower population density than Khulna, so there is more land available to be utilized for homestead production, and Khulna residents were more likely to live near periurban areas with markets, and therefore possibly less dependent on foods grown or raised in household gardens. Further, the soil in Khulna has higher levels of salinity which impedes crop growth. Regarding program implementation, SPRING believes that the FNS intervention was implemented consistently in both divisions, so the quality of implementation was not likely to be a cause for the differences observed. On the other hand, during the period when SPRING was implemented, there was another USAID-funded nutrition activity also operating in approximately six out of the 19 upazilas of Barisal. Therefore, it is possible that some participants in the cohort study also received knowledge and inputs from the other activity. The survey questionnaire did not ask respondents about exposure to other projects, so we cannot say with certainty whether or not that influenced our findings.

Program attendance also yielded differential results. WDDS of women who attended $100 \%$ of the sessions increased more from P1 to P2, than that attained among those who attended fewer than $75 \%$ of the sessions. At P1, the mean WDDS of women who would had attended $100 \%$ of the sessions was 3.8, lower than the WDDS of those who would had attended less than $75 \%$ of the sessions, which was 4.3 (difference barely reaching statistical difference). WDDS for these two groups significantly increased to 6.2 vs. 5.9 at P2 and stayed at 5.7 vs. 5.5 at P3. Although no statistical significance was found between the two groups at P2 and P3, the observed differential responses to interventions among women who had less than $75 \%$ of attendance versus those who attended all sessions suggested two possible lessons-learned for future programs: a) women who started with worse WDDS might have a stronger drive to take full advantage of the FNS program and demonstrated a strong ability/potential to benefit; $b$ ) there were still significant benefits for dietary diversity for those women who attended only $75 \%$ or less sessions. 
As an implementation project, SPRING paid attention to not only the technical details of our intervention but also to program learning. SPRING determined several elements that were key factors leading to successful FNS implementation and outcomes, and that could potentially be used when adapting the FNS approach in other contexts (Box 2) (SPRING 2017).

Some of the key factors are of particular importance. First, we worked closely with existing frontline workers affiliated with Ministry of Health and Family Welfare (MOHFW) and Ministry of Agriculture (MOA), instead of investing on setting up separate service providers and platforms. Second, our approach seems to support that a lighter touch (less intensive) program using locally available resources can have a significant effect on nutrition outcomes. Third, more specifically related to dietary diversity, locally sourced nutrient-rich foods need to be 'propped up' and promoted, to counter possible misconceptions that local foods are 'poor man's' foods and not as nutritious as the 'exotic' imported foods. Lastly, program implementers, and donors alike, should work with communities, as they are the source of sustainable changes. For instance, SPRING did not have a post-graduation plan for FNS

\section{Box 2. Key factors leading to successful FNS implementation and outcomes}

1. Capacity and ownership - Facilitators and other staff at all levels were supported by job aids and regular supervision. When the program ended, one of the FNS participants was nominated as the community nutrition "champion", and to participate in community clinic support groups at the community level. This formalization was key to long-term sustainability and maintaining a presence in the community.

2. Learning by doing. The FNS curriculum was designed and delivered as experiential learning with lots of time built in to practice hands-on skills in the field.

3. Consistent and repeated messaging. Behavior change requires reinforcement. The combination of consistency and repetition helped ensure that key messages were internalized and became common practice.

4. Minimal inputs. The FNS approach fostered an environment of independence and selfreliance, skills building, and peer-to-peer support mechanisms. There were no cash, inkind, or other financial incentives in FNS, except two distributions of seeds.

5. Low/no-cost solutions based on simple, evidence-based innovations. The program focused on what participants are already familiar with and could do with what they had. Two examples were 1) tippy taps, simple and cost-effective handwashing stations set up near cooking areas and latrines, and 2) improved hajols - hatching pots made of mud -for hens to get foods and water right next to them to preserve hens' energy and the temperature needed for the eggs, to boost egg production.

6. Whole family and community approach.

- SPRING engaged members of the family who might otherwise impede successful implementation of the program, such as husbands and mothers in law, by encouraging them to participate in various FNS activities.

- At the outset of a new FNS group, a community-wide "celebration" was held to allow members of the community to meet and understand what the FNS participants would learn about. At the end, a nutrition fair with awards was held.

- Community-wide buy-in was facilitated by framing participation as an educational opportunity to empower women to raise healthy, well-nourished children.

7. Mindfulness of women's time. Women in most rural contexts have a tremendous workload. Based on local contexts, no more than biweekly meetings were appropriate.

8. Season-based sessions. FNS sessions were designed to be relevant to the time of year and crop seasonality, to maximize year-round production of and access to diverse and nutritious foods. 
participants in the beginning of program. However, in those instances when we visited FNS graduates after they completed all sessions, we noticed that some women had taken it upon themselves to spread the messages they learned. It was because of this observation that SPRING institutionalized CNCs and the follow-up visits after the sessions ended. Therefore, we believe that it is likely that many homestead production and consumption outcomes achieved in our cohort would have been largely sustained after graduation based on FNS participants' individual motivation alone, though the formal establishment of CNCs and the few follow-up visits should have helped.

This study used a prospective cohort design spread across more than two divisions in Bangladesh, with only a small loss of follow-up in our samples over time. The study included consistent use of the survey instrument and enumerators in all three phases of the study, which increases the interval validity of data collected across time and space. One limitation of this cohort study was the lack of a comparison group. A rigorous stand-alone impact assessment was not considered possible, due to lack of resources and the fact that there were other larger-scale surveys taking place in the Feed the Future ZOI.

In order to assess the likelihood that the changes observed among FNS participants in the SPRING cohort study were actually due to FNS interventions, we referred to two national level population-based surveys (PBS) that have collected data in Barisal and Khulna in similar time periods. Specifically, we referenced data from the Food Security and Nutrition Surveillance Program (FSNSP),(Project 2014) a long-standing European Union-funded surveillance program, as well as from the Bangladesh Integrated Household Survey (BIHS),(IFPRI's Bangladesh Integrated Household Survey (BIHS) Second Round Dataset now available) (IFPRI, n.d.) funded by USAID, for proxy baseline and endline values. Results from both data sets did not indicate a significant secular trend of improved WDDS in the ZOI.

The first PBS we analyzed was a subset of survey rounds collected by FSNSP and a follow-on survey mechanism that used the same design and survey protocols as FSNSP. For a proxy baseline, we used three FSNSP rounds that were carried out in Barisal and Khulna between October 2011 and August 2012 (before SPRING's programmatic interventions began). For the proxy endline, we used three rounds conducted between February and December 2017 by the follow-on survey mechanism using the same questionnaire and methods. Both proxy baseline and endline data sets contained upazilas where SPRING operated and upazilas where we did not. We analyzed a subset of women sampled in these arounds, who most closely matched the SPRING FNS participants, namely, women of reproductive age with children less than two years of age and from households belonging to the poorest two wealth quintiles. A total of 629 and 439 women met these criteria for baseline and endline respectively, from of 26 upazilas, including 15 SPRING upazilas and 11 non-SPRING upazilas. Our data analysis showed that WDDS in SPRING upazilas increased slightly from 3.7 to 3.8 and declined slightly in non-SPRING upazilas (3.6 to 3.5), indicating lack of change in the overall population.(SPRING, forthcoming). 
Second, we looked at WDDS data among women of 15-49 years of age from BIHS in 2011-12 and 2015. BIHS is statistically representative at the following levels: (a) nationally representative of rural Bangladesh; (b) representative of rural areas of each of the seven administrative divisions of the country; and (c) representative of the Feed the Future ZOI. WDDS for both Barisal and Khulna increased, but only slightly, between the two rounds of BIHS datasets. WDDS for Barisal increased from $4.21 \pm 1.17$ in 2011-12 to $4.51 \pm 1.18$ in 2015, and WDDS for Khulna increased from $4.37 \pm 0.91$ in 2011-12 to $4.49 \pm 0.90$ in 2015. We speculate that WDDS' are higher among women surveyed by BIHS because the sample included households in all wealth quintiles, while the FNS participants were drawn from the two lowest wealth quintiles.

Analysis of both PBSs suggested that WDDS was remaining steady or increasing only slightly in the overall population of women with young children in Barisal and Khulna during the period of SPRING implementation, suggesting that the sizable and significant improvements in WDDS among FNS participants were unlikely due purely to chance.

The 2-stage sampling design resulted in the best combined effect of capturing the outcomes of our interventions. Our approach was able to reach a high level of precision by having FNS participants sampled from 38 out of 40 upazilas in which SPRING implemented FNS programs, meaning wider representation of the entire SPRING program area. There was some clustering effect since we sampled 10 women from the one (or two in a few cases) FNS that was selected within each of the upazilas. However, had we performed a complete simple random sampling of all FNS beneficiaries across the 40 upazilas, the expenses would have been prohibitive and we would have not been able to justify the cost for the additional precision gained.

Other limitations of the study include seasonal variations and reporting bias. P1 and P2 data collection was conducted at different times of the year since each FNS group lasted for nine months, which might have resulted in some of the observed changes, such as consumption of vitamin A-rich fruits and vegetables, which tend to be more readily available during the monsoon months, when P2 and P3 data were collected. However, during follow-up monitoring visits between P2 and P3, we observed that gardens appeared to be kept up throughout the year.

Since most responses recorded during the survey were self-reported, this study may also suffer from some level of social desirability bias and recall bias, as the FNS participants could have reported positive results to obtain approval from the project and enumerators. We used standard best practice survey techniques, however, and do not believe that such biases would be greater in this study than similar household nutrition surveys.

As for global implication of our program, we would like to emphasize that FNS was very successful in improving production and consumption diversity among a limited group of women in communities in which we worked in southern Bangladesh for a number of reasons. These included a thoughtful and adaptive management approach used by staff and leadership as well as good formative research at the outset that helped the team to understand and program to overcome constraints to engaging women with the range of FNS interventions. While the components of FNS - Farmer Field Schools, Homestead Food Production and ENA/EHA - are used in or adaptable to most settings, the ability we had to combine these to produce a diversity of nutrient-rich foods throughout most of the year in our target area is not something that can be 
assumed to be possible in all locations. Reliable water access is one of well-documented constraints for programs that aim to produce nutrient-rich foods year around. Other components of the Bangladesh context that contributed to improved dietary diversity of women through household food production included: a) Close proximity of women's homes combined with high population density making it possible to develop and maintain strong social cohesion among group members between meeting dates; b) Unique agro-ecological environment supporting opportunities for fish farming as well as poultry production ensuring access to at least one source of animal source food for nearly all FNS beneficiaries; and, c) Strong local NGO partners with adequate numbers of well-trained staff ensuring high quality training, follow-up and referral services embedded within the project.

The FNS approach focused on the pathway from production to consumption. The FNS did not assist women beneficiaries to sell the foods they grew because the focus was very much on encouraging the consumption of diverse, nutrient-rich foods they were growing or raising. However, staff recognized that as production skills grew, women were, in fact, selling some portion of their production and finding themselves in a position to control the income they were making. This was an unexpected, positive outcome for women's empowerment. Therefore, we believe that with additional time, the FNS could have been adapted to specifically promote marketing skills to maximize earnings while also promoting household budgeting and planning to engage men and other influencers together with women in sharing decisions on investment of income toward food and non-food purchases that will promote good nutrition. At present, there is a need for more evidence on the pathway from income to purchase (foods and non-foods) to improved nutrition outcomes. And, with foresight, the FNS approach could provide an excellent means for testing this.

\section{Conclusions}

While this cohort study did not have a built-in control group, the magnitude of the increases in production outcomes and WDDS among FNS participants, coupled with much smaller or no increases at the population level over even longer time periods, suggested that these results were at least partly due to the SPRING FNS intervention. The FNS approach, which supports agriculture interventions with an explicit nutrition objective, generated rapid and sustained improvement in food production and dietary quality. In summary, this study suggests that a compact program design based on local contexts, with moderate management support and minimum material inputs can have a powerful effect on nutrition outcomes within a short time, and, with community involvement and ownership in a context like Bangladesh, the improvements can be sustained.

Further research on FNS should explore the mediating effect of the uptake of innovative and low-cost agriculture production techniques, nutrition and hygiene knowledge on production and dietary outcomes in a more rigorous design, including a comparison group and longer follow up period if possible. A longer follow up period could also test the extent to which and at what point increased homestead production of nutrient-rich foods interacts with markets, and the evidence on the pathway from income to purchase (foods and non-foods) to improved nutrition outcomes thereby contributing to the global evidence on nutrition-sensitive agriculture programming. 


\section{References}

Braun, Arnoud and Deborah Duveskog. 2008. "The Farmer Field School Approach: History, Global Assessment and Success Stories.” Background Paper for the IFAD Rural Poverty Report, 43. http://www.ifad.int/rural/rpr2008/chapter3/3.pdf.

CoreGroup. 2015. "The Essential Nutrition Actions and Essential Hygiene Actions Training Guide: Health.” Accessed May 8, 2018. https://coregroup.org/wp-content/uploads/mediabackup/documents/Resources/Tools/ENA_EHA/ENAEHAFlyer_updated.pdf.

DHS Bangladesh. 2011. "Bangladesh Demographic and Health Survey 2014.” Journal of Mathematical Biology. Vol. 59. https://doi.org/10.1007/s00285-008-0246-3.

_. 20113. “Bangladesh Demographic and Health Survey 2011.” NIPORT, GoB. Vol. 59. https://doi.org/10.1007/s00285-008-0246-3.

Doocy Shannon, Sarah Cohen, Jillian Emerson, Joseph Menakuntuala, the Jenga Jamaa II Study Team, and Jozimo Santos Rocha. 2017. "Food Security and Nutrition Outcomes of Farmer Field Schools in Eastern Democratic Republic of the Congo.” Global Health: Science and Practice 5 (4):630-43. https://doi.org/10.9745/GHSP-D-17-00203.

Dulal Bishnu, Gary Mundy, Rojee Sawal, Pooja Pandey Rana, and Kenda Cunningham. 2017. "Homestead Food Production and Maternal and Child Dietary Diversity in Nepal: Variations in Association by Season and Agroecological Zone.” Food and Nutrition Bulletin 38 (3):338-53. https://doi.org/10.1177/0379572117703264.

FAO. 2010. Guidelines for Measuring Household and Individual Dietary Diversity. FAO. - 2018. "Farmer Field School Approach.” FAO. 2018. http://www.fao.org/agriculture/ippm/programme/ffs-approach/en/.

FAO and FHI 360. 2016. "Minimum Dietary Diversity for Women- A Guide to Measurement." Rome. http://www.fao.org/3/a-i5486e.pdf.

Girard Amy Webb, Julie L. Self, Corey McAuliffe, and Olafunke Olude. 2012. “The Effects of Household Food Production Strategies on the Health and Nutrition Outcomes of Women and Young Children: A Systematic Review.” Paediatric and Perinatal Epidemiology 26 (July):205-22. https://doi.org/10.1111/j.1365-3016.2012.01282.x.

Harris-Fry Helen, Kishwar Azad, Abdul Kuddus, Sanjit Shaha, Badrun Nahar, Munir Hossen, Leila Younes, Anthony Costello, and Edward Fottrell. 2015. “Socio-Economic Determinants of Household Food Security and Women's Dietary Diversity in Rural Bangladesh: A Cross-Sectional Study.” Journal of Health, Population and Nutrition 33 (1):2. https://doi.org/10.1186/s41043-015-0022-0.

Iannotti Lora, K Cunningham, and M Ruel. 2009. "Improving Diet Quality and Micronutrient Nutrition: Homestead Food Production in Bangladesh.” International Food Policy Research Institute.

“IFPRI’s Bangladesh Integrated Household Survey (BIHS) Second Round Dataset Now Available | IFPRI.” n.d. Accessed April 5, 2018. http://www.ifpri.org/blog/ifprisbangladesh-integrated-household-survey-bihs-second-round-dataset-now-available.

Jones Andrew D. 2017. "Critical Review of the Emerging Research Evidence on Agricultural Biodiversity, Diet Diversity, and Nutritional Status in Low- and Middle-Income Countries.” Nutrition Reviews 75 (10):769-82. https://doi.org/10.1093/nutrit/nux040. 
Kuchenbecker Judith, Anika Reinbott, Beatrice Mtimuni, Michael B. Krawinkel, and Irmgard Jordan. 2017. "Nutrition Education Improves Dietary Diversity of Children 6-23 Months at Community-Level: Results from a Cluster Randomized Controlled Trial in Malawi.” Edited by Jacobus P. van Wouwe. PLOS ONE 12 (4). Public Library of Science:e0175216. https://doi.org/10.1371/journal.pone.0175216.

Olney D. K., L. Bliznashka, A. Pedehombga, A. Dillon, M. T. Ruel, and J. Heckert. 2016. “A 2Year Integrated Agriculture and Nutrition Program Targeted to Mothers of Young Children in Burkina Faso Reduces Underweight among Mothers and Increases Their Empowerment: A Cluster-Randomized Controlled Trial.” Journal of Nutrition 146 (5):1109-17. https://doi.org/10.3945/jn.115.224261.

Food Security Nutritional Surveillance. 2014. "Food Security Nutritional Surveillance Project.” 2014.

Reinbott Anika, Anna Schelling, Judith Kuchenbecker, Theresa Jeremias, Iean Russell, Ou Kevanna, Michael B. Krawinkel, and Irmgard Jordan. 2016. "Nutrition Education Linked to Agricultural Interventions Improved Child Dietary Diversity in Rural Cambodia.” British Journal of Nutrition 116 (8). Cambridge University Press:1457-68. https://doi.org/10.1017/S0007114516003433.

Ruel, Marie T. 2003. "Is Dietary Diversity an Indicator of Food Security or Dietary Quality? A Review of Measurement Issues and Research Needs.” Food and Nutrition Bulletin 24 (2):231-32. https://doi.org/10.1177/156482650302400210.

Ruel, Marie T., and Harold Alderman. 2013. "Nutrition-Sensitive Interventions and Programmes: How Can They Help to Accelerate Progress in Improving Maternal and Child Nutrition?” The Lancet 382 (9891):536-51. https://doi.org/10.1016/S0140-6736(13)60843-0.

Shamim Abu Ahmed, Saidur Rahman Mashreky, Tarana Ferdous, Kathrin Tegenfeldt, Sumitro Roy, A. K.M.Fazlur Rahman, Iftekhar Rashid, et al. 2016. "Pregnant Women Diet Quality and Its Sociodemographic Determinants in Southwestern Bangladesh.” Food and Nutrition Bulletin 37 (1):14-26. https://doi.org/10.1177/0379572116632137.

Sinharoy Sheela S., Jillian L. Waid, Regine Haardörfer, Amanda Wendt, Sabine Gabrysch, and Kathryn M. Yount. 2018. "Women’s Dietary Diversity in Rural Bangladesh: Pathways through Women’s Empowerment.” Maternal \& Child Nutrition 14 (1):e12489. https://doi.org/10.1111/mcn.12489.

SPRING. forthcoming. “Trends in Homestead Food Production and Nutrition Outcomes in the Feed the Future Zone of Influence, Bangladesh: An Impact Assessment of SPRING Interventions from 2012 - 2016.” Arlington, VA.

- 2017. "Farmer Nutrition School Advocacy Guide with Recommendations for Adaptation: SPRING/Bangladesh.” Arlington, VA. https://www.springnutrition.org/sites/default/files/publications/tools/spring_bd_farmer_nutrition_advocacy.pdf. USAID. 2010. “Feed the Future Guide.” Framework May:35. https://doi.org/10.1136/ip.2008.019778.

WHO. 2013. “Essential Nutrition Actions.” Essential Nutirittion Actions, 144. 